
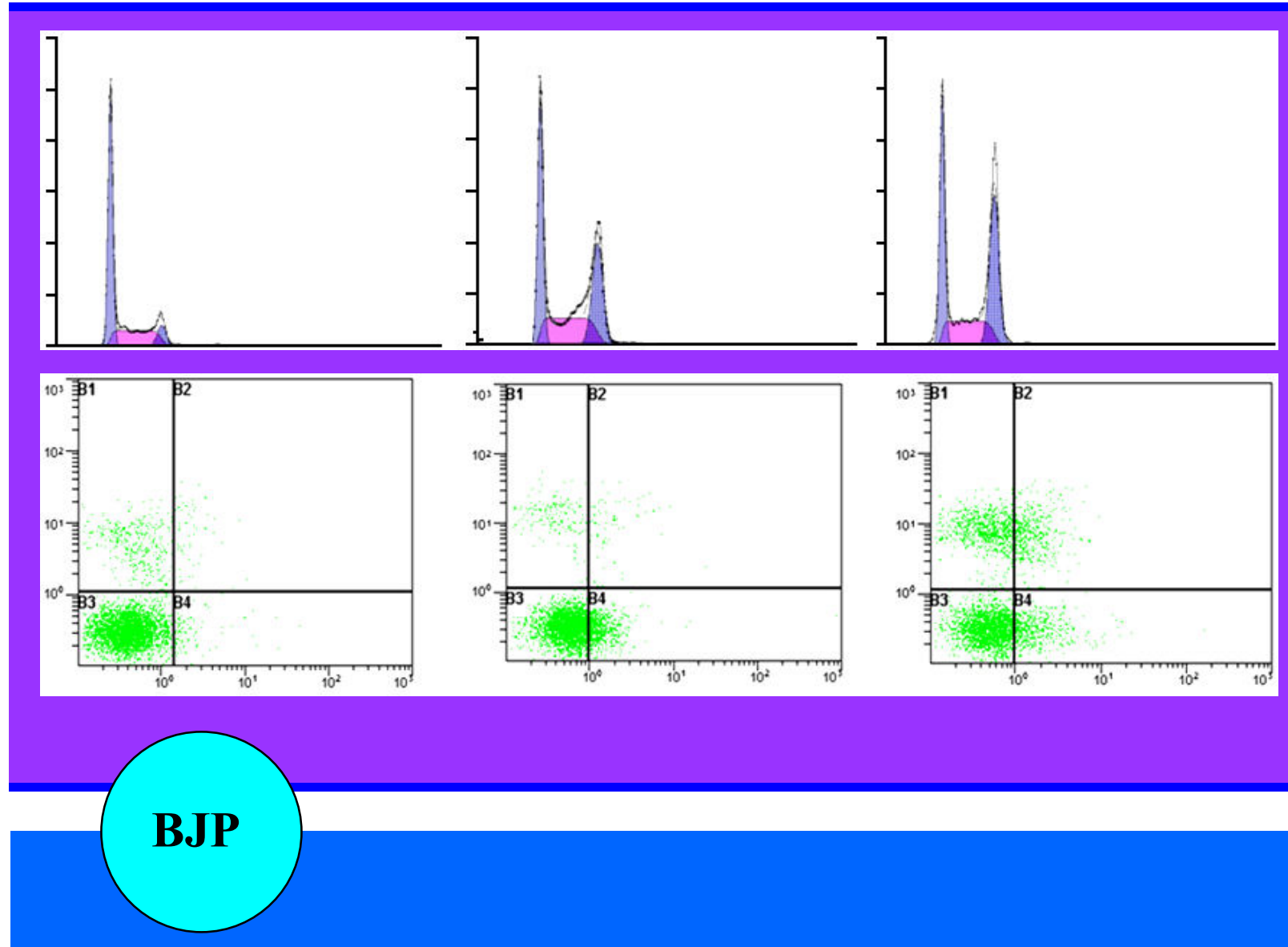

Bangladesh Journal of Pharmacology

Research Article

\title{
Jaceosidin inhibits proliferation of human bladder cancer T24 cells through induction of cell cycle arrest and apoptosis
}




\title{
Jaceosidin inhibits proliferation of human bladder cancer T24 cells through induction of cell cycle arrest and apoptosis
}

\author{
Yong $\mathrm{Li}^{1,2}$ and Yan $\operatorname{Tan}^{1,3}$ \\ ${ }^{1}$ The first Hospital of Jilin University, Changchun 130021, China; ${ }^{2}$ The $210^{\text {th }}$ Hospital of PLA, Dalian 116100, China; \\ ${ }^{3}$ The People's Hospital of Jilin Province, Changchun 130021, China.
}

\begin{tabular}{|c|c|}
\hline \multicolumn{2}{|l|}{ Article Info } \\
\hline Received: & 7 July 2013 \\
\hline Accepted: & 29 July 2013 \\
\hline Available Online: & 21 August 2013 \\
\hline \multicolumn{2}{|c|}{ DOI: 10.3329/bjp.v8i3.15620 } \\
\hline \multicolumn{2}{|c|}{$\begin{array}{l}\text { Cite this article: } \\
\text { Li Y, Tan Y. Jaceosidin inhibits prolif- } \\
\text { eration of human bladder cancer T24 } \\
\text { cells through induction of cell cycle } \\
\text { arrest and apoptosis. Bangladesh J } \\
\text { Pharmacol. 2013; 8: 349-56. }\end{array}$} \\
\hline
\end{tabular}

\begin{abstract}
Jaceosidin, isolated from Artemesia argyi, has been shown to possess promising anti-cancer potential against various cancer cells. However, its effect against bladder cancer cells remained unknown. In this study, for the first time, we investigated the effects of jaceosidin on cell proliferation, cell cycle, and apoptosis in bladder cancer T24 cells by using MTT assay and flow cytometric analysis. The results revealed that jaceosidin decreased the cell viability of bladder cancer T24 cells in a dose- and time-dependent manner. Flow cytometric analysis demonstrated that jaceosidin significantly triggered apoptosis in T24 cells and arrested cell cycle at G2/M phase in a timedependent manner. Further characterization showed that jaceosidin-induced apoptosis is associated with dissipation in mitochondrial membrane potential $\left(\Delta \Psi_{\mathrm{m}}\right)$, up-regulation of Bax and down-regulation of Bcl-2 in jaceosidintreated T24 cells. These in vitro results suggested that jaceosidin should be further examined for in vivo activity and molecular mechanism in human bladder cancer.
\end{abstract}

\section{Introduction}

Natural products have become one of the most imperative sources of prospective anticancer agents and several compounds, which were originally isolated from plants such as paclitaxel, camptothecin, vinca alkaloids, and etoposide have potential applications in cancer chemotherapy, therefore, plants are considered as one of the most important sources for the development of novel anti-cancer drugs (Amin et al., 2009; Cragg and Newman, 2005). Artemisia plants possess a variety of biological activities and draw considerable attention in pharmacological research due to their anti-inflammatory, anti-malarial, anti-hepatitis, and anti-cancer activities (Khan et al., 2012; Lee et al., 2003; Tan et al., 1998). Jaceosidin (Figure 1), isolated from Artemesia argyi, has been reported to have promising biological effects, including anti-tumorigenic, antioxidant, and anti-inflammatory activities (Khan et al.,
2012; Kim et al., 2008; Min et al., 2009). Furthermore, jaceosidin has anti-proliferative effect and induces apoptosis in various cancer cells such as endometrial cancer cells (Lee et al., 2013), ovarian cancer cells (Lv et al., 2008), and glioblastoma U87 cells (Khan et al., 2011;

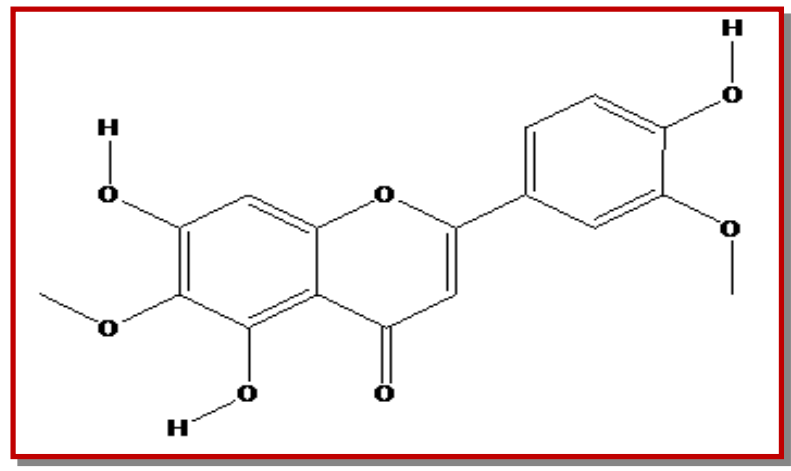

Figure 1: Structure of jaceosidin 
Khan et al., 2012). However, the cytotoxic effects of jaceosidin on bladder cancer and its mechanism were still unknown.

Bladder cancer is an increasingly common and potentially lethal malignancy (Ploeg et al., 2009). Bladder cancer treatment is widely studied by single and combination of anti-cancer drugs (Konno, 2007). These chemotherapeutic treatments were not potent enough against bladder cancer and development of novel compounds that can prevent proliferation of bladder cancer is in progress (Konno, 2007; Ploeg et al., 2009). In the last few decades, several natural compounds have shown a great promise in treatment of cancer and prevention of metastasis; most of the researchers are interested in and are focusing on finding a cure for cancer and several published reports have identified numerous dietary and botanical natural compounds that have chemotherapeutic potential (Rasul et al., 2013). Therefore, novel therapeutic agents and treatment approaches are desired to improve the clinical outcome. For this purpose, natural compounds were screened against human bladder cancer. Jaceosidin was one of them which showed potent antiproliferative effect against T24 cells. Further characterization showed that jaceosidin effectively inhibited the proliferation of T24 cells through arresting cell cycle at G2/M phase and induction of apoptosis which is regulated by downregulation of $\mathrm{Bcl}-2$ and up-regulation of Bax.

\section{Materials and Methods}

\section{Chemicals and reagents}

Cell culture medium reagents and MTT [3'-(4, 5 dimethyl-thiazol-2-yl)-2, 5-diphenyl tetrazolium bromide], propidium iodide (PI), and dimethyl sulfoxide (DMSO) were purchased from Sigma. Fetal bovine serum (FBS) was purchased from the Hangzhou Sijiqing Biological Engineering Materials Co., Ltd. Annexin V-FITC apoptosis detection kit was purchased from Beyotime Institute of Biotechnology Shanghai, China. Rabbit polyclonal anti-human Bcl-2 and Bax antibodies were purchased from Wuhan Boster Biological Technology Co., Ltd. $\beta$-actin, anti-mouse and anti-rabbit antibodies were purchased from Santa Cruz Biotechnology. Ponceou and cell lysis buffer for Western and IP were purchased from Bio SS Beijing. Rhodamine 123 was purchased from Eugene Co. (USA).

\section{Cell culture}

Human bladder cancer T24 cells were propagated in DMEM nutrients mixture supplemented with 10\% FBS and antibiotics at $37^{\circ} \mathrm{C}$ in a humidified atmosphere with $5 \% \mathrm{CO}_{2}$ and $95 \%$ air. Cells were seeded in $10 \mathrm{~cm}$ culture dish and allowed to grow to approximately $70 \%$ confluence before experimentation.

\section{Cell proliferation assay}

The cytotoxic effects of the jaceosidin on the cells were determined by MTT assay as described previously (Rasul et al., 2011). Briefly, T24 cells were seeded at a density of $1 \times 10^{4}$ cells per well in 96-well plates and were allowed to grow overnight. Cells were incubated with $100 \mu \mathrm{L}$ of complete culture medium containing 0 , 30,100 and $300 \mu \mathrm{M}$ of jaceosidin. After incubation for 24 and 48 hours, growth of cells was determined by adding $10 \mu \mathrm{L}$ MTT $(5 \mathrm{mg} / \mathrm{mL}$ in phosphate buffered saline) to each well and incubated for 4 hours. After removal of the medium, $150 \mu \mathrm{L}$ DMSO was added to each well and shaken carefully. The absorbance was read at a wavelength of $570 \mathrm{~nm}$ in a plate reader (ELX 800, BIO-TEK Instruments Inc.). The growth curve was plotted against mean values which were calculated using the following equation:

$\mathrm{I} \%=\left[\mathrm{A}_{570}(\right.$ control $)-\mathrm{A}_{570}($ treated$\left.)\right] / \mathrm{A}_{570}($ control $) \times 100$

\section{Flow cytometric analysis of cell cycle}

For cell analysis, T24 cells were seeded in 12-well plates and then treated with $100 \mu \mathrm{M}$ of jaceosidin for 24 and 48 hours. After treatments, the percentages of cells in the different phases of cell cycle were evaluated by determining the DNA contents after propidium iodide (PI) staining as described somewhere else (Rasul et al., 2012b). Briefly, cells were washed with PBS, trypsinized and centrifuged at $1,000 \mathrm{rpm}$ at $4^{\circ} \mathrm{C}$ for $5 \mathrm{~min}$. Pellets were fixed overnight in $70 \%$ cold ethanol. After fixation, cells were washed twice with PBS and incubated in PBS containing RNase $(1 \mathrm{mg} / \mathrm{mL})$ for 10 $\mathrm{min}$ at room temperature. Finally, samples were stained with propidium iodide $(1 \mathrm{mg} / \mathrm{mL})$ for $30 \mathrm{~min}$ at $4^{\circ} \mathrm{C}$. Data acquisition was done by flow cytometry (EPICSXL -MCL, Beckman Coulter, US) using Cell Quest software.

\section{Flow cytometric determination of apoptosis}

The apoptotic rate of T24 cells were examined by flow cytometry using annexin V-FITC/PI staining. Briefly, T24 cells were cultured in 6-well plates and allowed to attach overnight. Cells were treated with $100 \mu \mathrm{M}$ of jaceosidin for 24 and 48 hours. Then cells were collected, washed and re-suspended in PBS. Apoptotic cell death was measured by double staining annexin $\mathrm{V}$ FITC and PI using the Annexin V-FITC apoptosis detection kit (Beyotime Biotechnology Shanghai, China) according to the manufacturer's instructions. Flow cytometric analysis was performed immediately after staining. Data acquisition and analysis were performed by flow cytometry using Cell Quest software.

\section{Flow cytometric determination of mitochondrial mem- brane potential $\left(\Delta \Psi_{m}\right)$}

To probe the changes in $\Delta \Psi_{\mathrm{m}}, \mathrm{T} 24$ cells were stained with Rhodamine-123 $(1 \mu \mathrm{M})$ after treatment of $100 \mu \mathrm{M}$ of jaceosidin for 24 and 48 hours with control group. 
The fluorescence of rhodamine- 123 was measured by flow cytometry with excitation and emission wavelengths of 488 and $530 \mathrm{~nm}$.

\section{Western blotting}

To reveal the mechanism of the apoptotic effect of jaceosidin, Western blotting was performed for apoptotic related proteins as previously described (Rasul et al., 2012a). Briefly, T24 cells were incubated with $100 \mu \mathrm{M}$ of jaceosidin for indicated time. Cells were trypsinized, collected in $1.5 \mathrm{~mL}$ centrifuge tube and washed with PBS. The cell pellets were resuspended in lysis buffer and were lysed on ice for $30 \mathrm{~min}$. After centrifugation for $15 \mathrm{~min}$, the supernatant fluids were collected and the protein content of the supernatant was measured by the NanoDrop 1000 spectrophotometer (Thermo Scientific, USA). The protein lysates were separated by electrophoresis on $12 \%$ SDS-polyacrylamide gel and transferred to a PVDF membrane (Amersham Biosciences, USA). The membranes were soaked in blocking buffer $(5 \%$ skimmed milk) for 2 hours. To probe for Bcl-2, Bax, and $\beta$-actin; membranes were incubated overnight at $4^{\circ} \mathrm{C}$ with relevant antibodies, followed by appropriate HRP conjugated secondary antibodies and ECL detection.

\section{Statistical analysis of data}

For the statistical analysis of data, comparisons between results from different groups were analyzed with SPSS for Window Version 15.0. Student's t-test was employed to determine the statistical significance of the difference between different experimental groups and control group. $\mathrm{p}<0.05$ value was defined as statistically

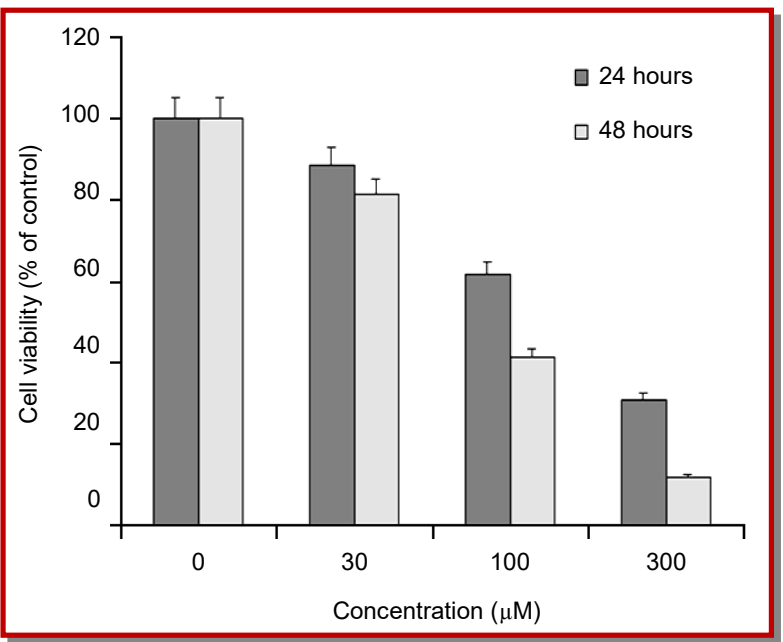

Figure 2: Jaceosidin inhibited the cell proliferation and induced cell death

T24 Cells were treated with indicated doses of jaceosidin for 24 and 48 hours and cell viability was measured by MTT assay. The effect of jaceosidin on the cell growth inhibition of T24 cells compared with control. The graph was plotted against mean values of percentages of three independent experiments. Data are expressed as Mean \pm SD ( $n=$ 3) significant. All experiments were repeated at least three times. Data were presented as mean \pm standard deviation (SD).

\section{Results and Discussion}

The investigation was started with screening of natural compounds against T24 human bladder cancer cells. We found that jaceosidin effectively exhibited cytotoxic effects on the growth of T24 cells. Jaceosidin is a natural compound that belongs to a flavonoid family. The effects of jaceosidin were examined on the growth of T24 human bladder cancer cells by quantifying the viable cells using MTT assay. Jaceosidin inhibited the growth of T24 human bladder cancer cells in a doseand time-dependent manner (Figure 2). Morphological changes were observed under phase contrast microscopy after treating cells with $100 \mu \mathrm{M}$ of jaceosidin for 24 and 48 hours. There was a significant decrease in the number of jaceosidin-treated T24 cells as compared to the untreated cells. Furthermore, jaceosidin-treated T24 cells become round-shaped and poorly adhered to the cultured plates while the untreated cells showed a typical polygonal and cobblestone monolayer appearance and remained firmly attached to cultured plates (data not shown). The results indicated that jaceosidin induced growth inhibition of T24 cells, in addition to other type of cancer cells previously reported including endometrial cancer (Lee et al., 2013), ovarian cancer (Lv et al., 2008), and glioblastoma U87 cells (Khan et al., 2011; Khan et al., 2012).

Recent insights related to cell cycle regulation indicated that there are a number of mechanisms which control the cell cycle to ensure the correct cell division. It is well known that progression of cell cycle is maintained by different check points in normal cells and the transition from one cell cycle phase to another occurs in an orderly fashion. In cancerous cells, some basic alterations transpired in the genetic control of cell division, resulting in a hyper cell proliferation. As the deregulation of cell cycle progression is the hallmark of cancer; thereby cell cycle regulation could be a potential and effective strategy for the treatment of cancer (Grana and Reddy, 1995; Vermeulen et al., 2003). Previous studies have shown that pharmacological correction of these check points and proper progression of cell cycle is a proficient strategy to control the growth and proliferation of cancer cells (Khan et al., 2012; Rasul et al., 2012a; Rasul et al., 2012b). Therefore, we analyzed effect on cell cycle progression of T24 cells. These findings reveal that jaceosidin arrested cell cycle at G2/M phase, which are in line with previously reported results in human glioblastoma U87 cells (Khan et al., 2011). The percentage of accumulation of cells in the G2/M phase was increased from $15.0 \pm 1.0 \%$ in untreated cells to 25.6 $\pm 3.2 \%$, and $36.4 \pm 2.9 \%$ in the cells treated with $100 \mu \mathrm{M}$ 
of jaceosidin for 24 and 48 hours respectively. This increase was coupled with the decreased percentage of cells in G0/G1 phase (Figure 3). These results revealed that G2/M phase cell cycle arrest was one of the mechanisms through which jaceosidin induces cytotoxicity in T24 cells. A number of recent studies have shown that by arresting the cell division at certain checkpoints in the cell cycle, several chemotherapeutic agents have shown potential antiproliferative effects (Khan et al., 2012; Rasul et al., 2012a; Rasul et al., 2012b).

Apoptosis, autophagy, and necrosis are the major types of cell death (Leist and Jaattela, 2001). Among the three major pathways of cell death, apoptosis is most well planned and orderly mode of cell death (Elmore, 2007; Hengartner, 2000). More than 50\% of neoplasm's undergo aberrations in the apoptotic machinery which leads to abnormal cell proliferation (Mashima and Tsuruo, 2005; Pommier et al., 2004). The regulation of apoptosis is, therefore, the most important in the treatment of cancer (Fulda, 2010; Lawen, 2003; Reed, 2002). Accumulated evidences indicated that the most of chemotherapeutic agents halt tumor cells proliferation via induction of apoptosis (Saha et al., 2010; Wu et al., 2009; Zhang, 2002). We examined whether jaceosidin inhibited cell growth of T24 cells through the induction of apoptosis. Jaceosidin-induced apoptosis was examined by flow cytometric analysis. Cells were seeded in the 12 well plates. After incubation of cells with or without jaceosidin for 24 and 48 hours, cells were collected in centrifuged tubes and stained with annexin V-FITC and PI double staining as described in materials and methods. The results of flow cytometric analysis showed that the rates of apoptosis were $19.5 \pm 2.1$ and $32.7 \pm 3.7 \%$ in the cells treated with $100 \mu \mathrm{M}$ of jaceosidin for 24 and 48 hours respectively as compared to the 2.3 $\pm 0.9 \%$ in control cells (Figure 4 ). Jaceosidin-induced apoptosis in T24 cells were compatible with previously reported studies in various cancer cells, including endometrial cancer cells (Lee et al., 2013), ovarian cancer cells (Lv et al., 2008), and glioblastoma U87 cells

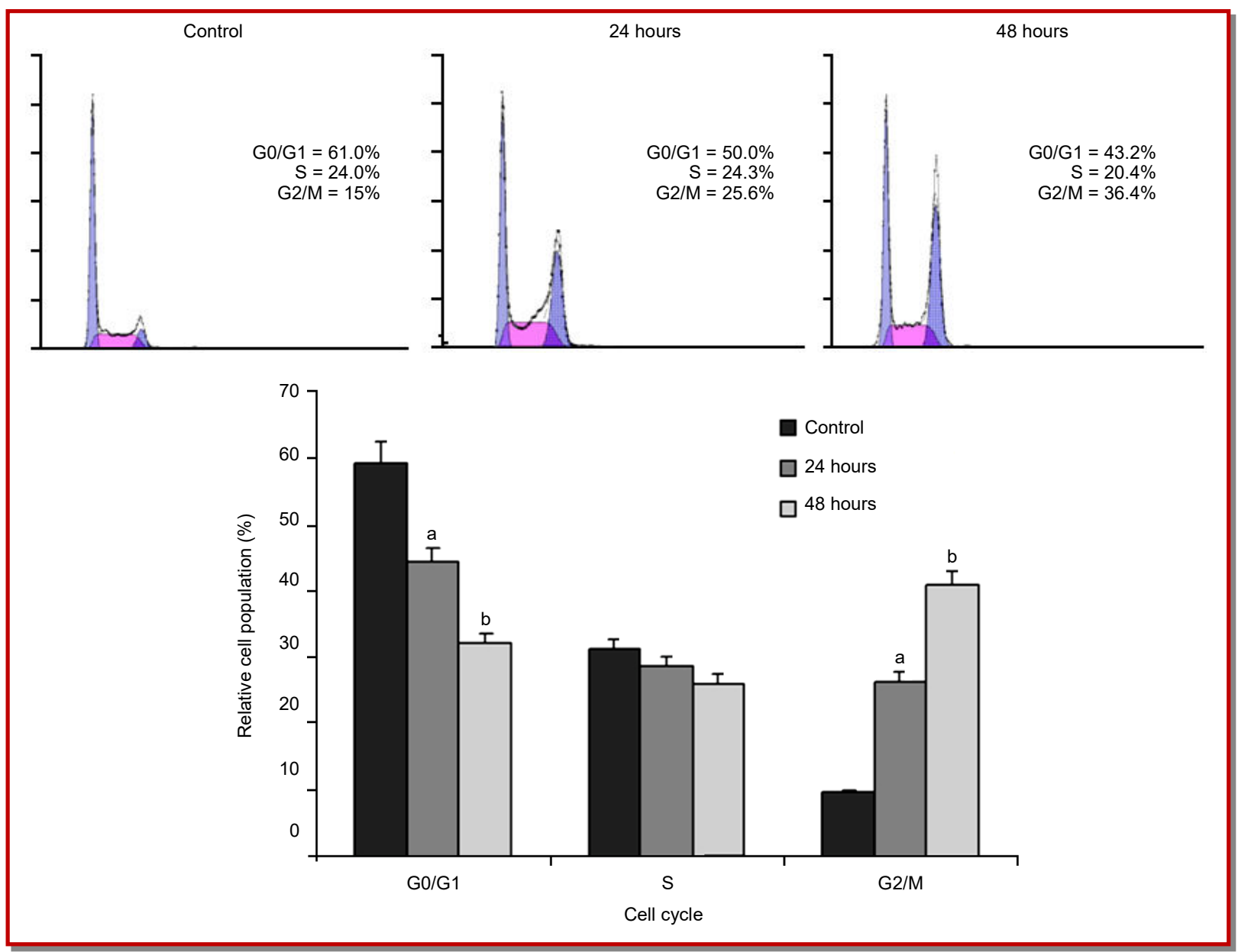

Figure 3: Effect of jaceosidin on cell cycle distribution.

T24 cells were treated with $100 \mu \mathrm{M}$ of jaceosidin for 24 and 48 hours and then stained with PI for flow cytometric analysis. Histograms show number of cells/channel (y-axis) vs. DNA contents ( $x$-axis). The values indicate the percentages of cells in the indicated phases of cell cycle. The data shown are representative of three independent experiments with the similar results. ${ }^{a} \mathrm{p}<0.05$ and ${ }^{b} \mathrm{p}<0.01$ compared with the control 
(Khan et al., 2012).

Many studies revealed that mitochondrion is one of the essential components of the apoptosis execution machinery, which contain pro-apoptotic proteins (e.g., cytochrome c) (Elmore, 2007). It has been elucidated that upon the depolarization of the mitochondrial membrane potential results in the mitochondrial swelling and subsequent release of cytochrome $c$ from the inter-mitochondrial membrane spaces into the cytosol (Buytaert et al., 2007). It is becoming increasingly apparent that mitochondria play a fundamental role in the processes those lead to the cell death (Wang, 2001). To probe the effect of jaceosidin on the $\Delta \Psi_{\mathrm{m}}, \mathrm{T} 24$ cells were stained with the Rho-123 to measure the mitochondrial trans-membrane potential. Effects of jaceosidin on the mitochondrial membrane potential of T24 cells were examined by flow cytometry using rhodamine-123 staining. The loss of mitochondrial membrane potential was considered directly proportional to the decrease of rhodamine- 123 fluores- cence in treated cells as compared to the normal cells. The rates of depletion of mitochondrial membrane potential were $83.7 \pm 2.9$ and $68.2 \pm 2.1 \%$ in the cells treated with $100 \mu \mathrm{M}$ of jaceosidin for 24 and 48 hours respectively as compared to $94.5 \pm 0.8 \%$ in control group (Figure 5). The results indicated the depletion of mitochondrial transmembrane potential by jaceosidin in a time-dependent manner in T24 cells. Our data corroborate with the previously reported results that aceosidin induced dissipation of mitochondrial membrane potential, which provide the evidence for direct contribution of mitochondria in the jaceosidin-induced apoptosis (Khan et al., 2012).

Interplay between pro-apoptotic (Bax) and antiapoptotic (Bcl-2) members of the Bcl-2 family pedals the mitochondrial apoptotic pathway (Mallat and Tedgui, 2000). Bcl-2 family proteins are pivotal for rising permeability of mitochondrial membranes and the release of cytochrome $c$, which activates caspases and in turn mobilizes apoptotic cell death (Adams and Cory,

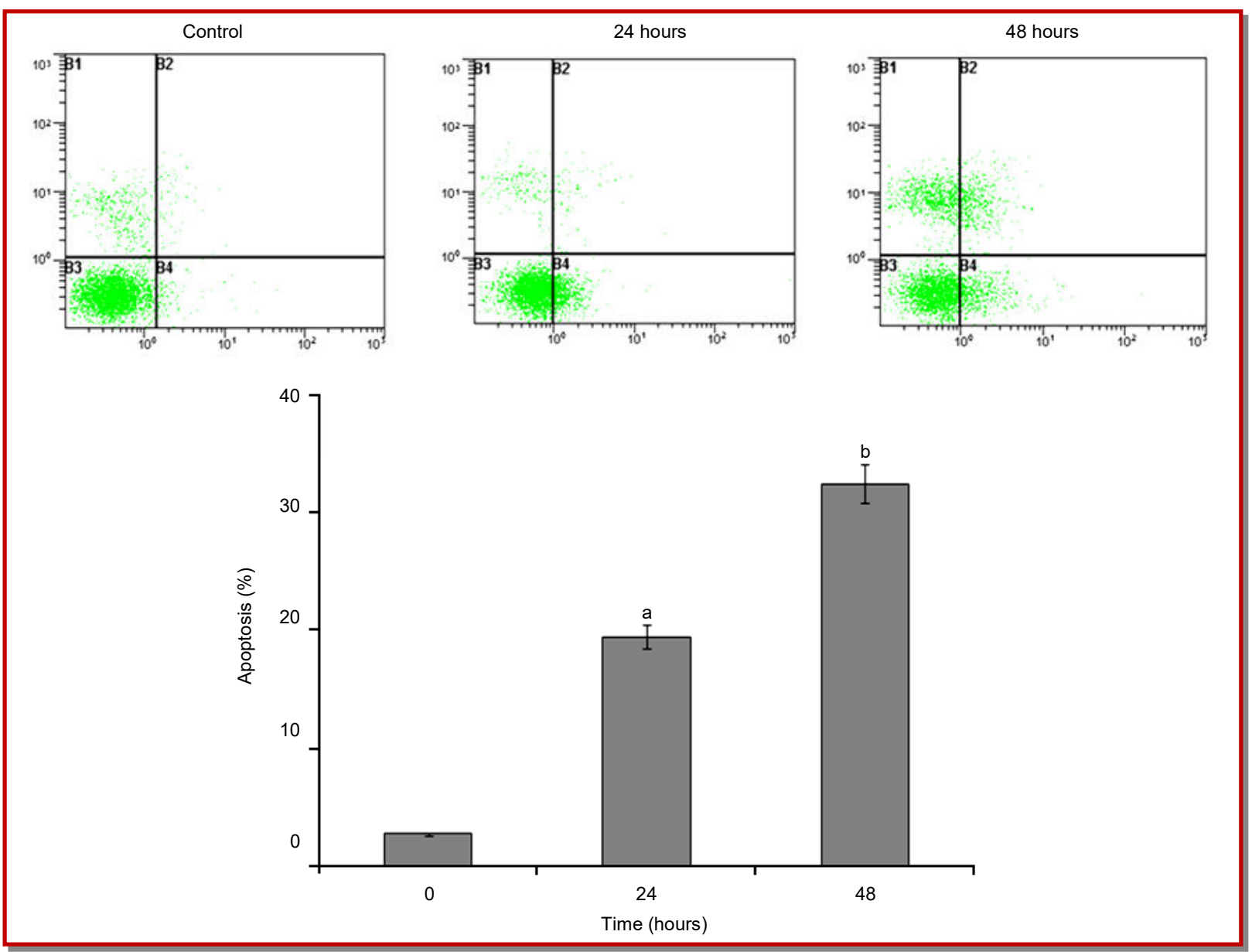

Figure 4: Apoptosis induced by jaceosidin in T24 cells

T24 cells were treated with $100 \mu \mathrm{M}$ of jaceosidin for 24 and 48 hours. Then cells were stained with FITC-conjugated Annexin V and PI for flow cytometric analysis. The flow cytometry profile represents Annexin V-FITC staining in $x$ axis and PI in $y$ axis. The number represents the percentages of apoptotic cells in each condition. The data shown are representative of three independent experiments with the similar results. ap $<0.05$ and bp $<0.01$ compared with the control 
2007; Burlacu, 2003; Danial, 2007). The release of cytochrome $c$ from the mitochondria into cytosol is coupled with the ratio of Bax/Bcl-2 proteins (Kluck et al., 1997). Bcl-2 family proteins are mostly involved in the mitochondrial apoptotic pathway (Reed, 1998). Furthermore, Bcl-2 family plays a central role in activation of caspases (Burlacu, 2003). Bcl-2 and Bax work in an antagonistic manner, Bcl-2 is anti-apoptotic while Bax is pro-apoptotic member of Bcl-2 family and Bcl-2 forms heterodimers with Bax. Bax inserts into outer membrane of mitochondria under stress conditions, as a result trans-membrane permeability become higher which facilitate the release of cytochrome $C$ due to formation of pores on the mitochondrial outer membrane (Adams and Cory, 2007; Danial, 2007). The balance between these two groups is critical in the cell decision to undergo apoptosis or not (Mallat and Tedgui, 2000). Therefore, we performed Western-blotting to examine the effects of jaceosidin on the expression of Bcl-2 and Bax in T24 cells. Jaceosidin was observed to be involved in the up-regulation of Bax and down regulation of $\mathrm{Bcl}-2$ in a time-dependent manner (Figure 6). These results are similar with previously reported studies in human glioblastoma U87 cells (Khan et al., 2012).

\section{Conclusion}

Jaceosidin induced apoptosis of T24 cells together with a significant loss of $\mathrm{G} 2 / \mathrm{M}$ phase cells. Jaceosidininduced apoptosis marked with down regulation of Bcl2 and up-regulation of Bax in a time-dependent manner. Moreover, this compound markedly induced a depolarization of mitochondrial membranes to facilitate cytochrome $c$ release into cytosol. The findings recommended that jaceosidin might be promising agent for treatment of cancer. These in vitro results suggested that jaceosidin should be further examined for in vivo activity and molecular mechanism in human bladder cancer.

\section{References}

Adams JM, Cory S. The Bcl-2 apoptotic switch in cancer development and therapy. Oncogene 2007; 26: 1324-37.

Amin AR, Kucuk O, Khuri FR, Shin DM. Perspectives for

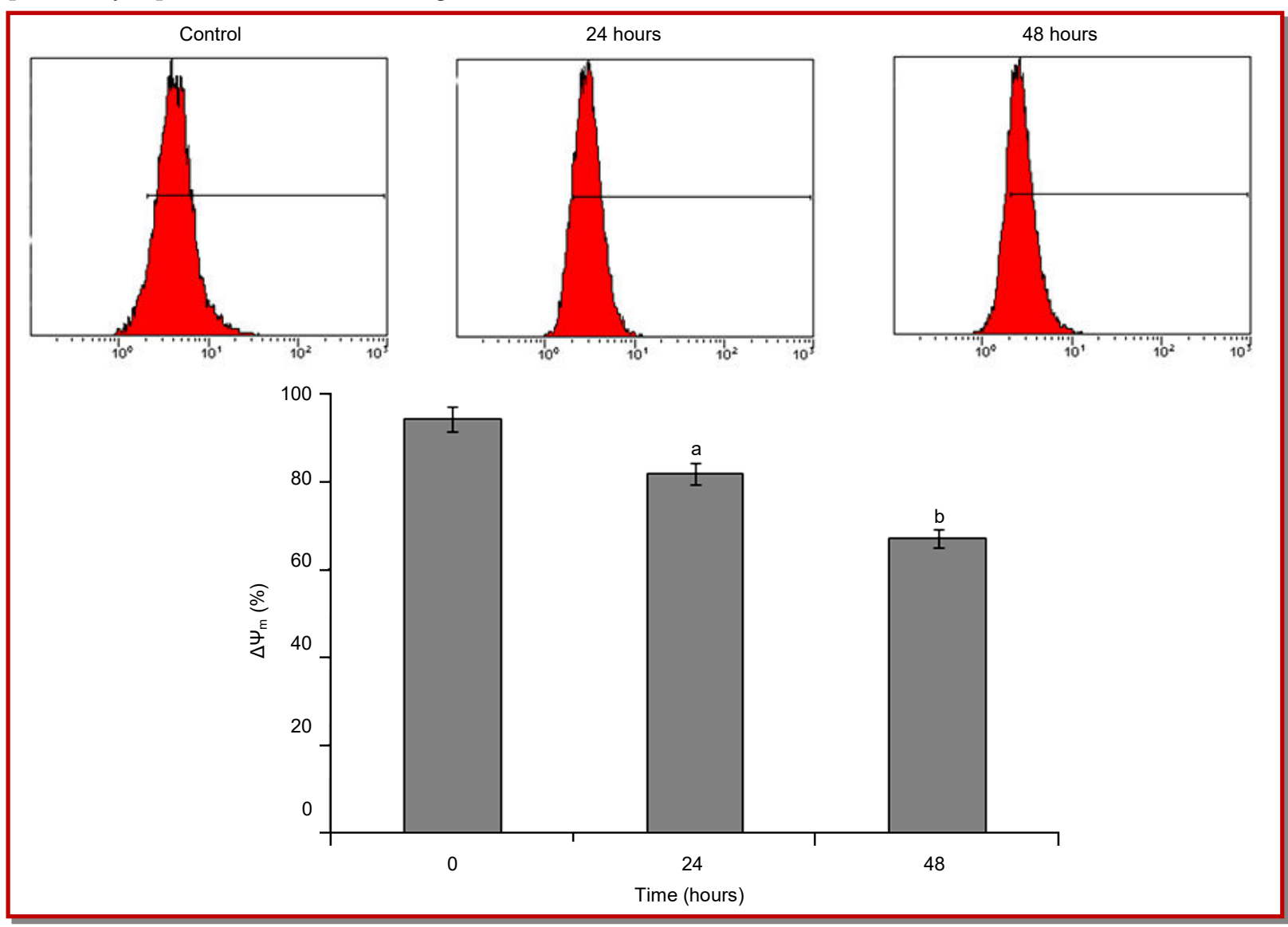

Figure 5: The effects of jaceosidin on mitochondrial transmembrane potential of T24 cells were determined by flow cytometry

The values indicate the percentages of rhodamine 123 fluorescence in the T24 cells treated with $100 \mu \mathrm{M}$ of jaceosidin or without (control) for 24 and 48 hours. The data shown are representative of three independent experiments with the similar results. ${ }^{a} p<0.05$ and bp $<0.01$ compared with the control 


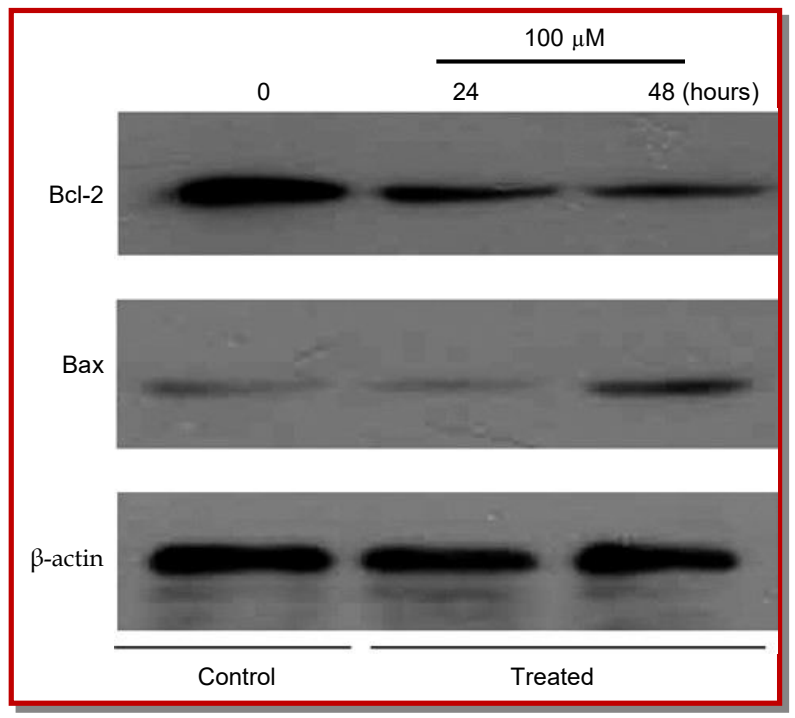

Figure 6: T24 cells were exposed to $100 \mu \mathrm{M}$ of jaceosidin for specified time intervals

Equal amounts of lysate protein were subjected to gel electrophoresis. Expression levels of Bcl-2 and Bax were monitored by Western-blot assay. $\beta$-actin was used as loading control. Data are representative of at least two independent experiments with similar results

cancer prevention with natural compounds. J Clin Oncol. 2009; 27: 2712-25.

Burlacu A. Regulation of apoptosis by Bcl-2 family proteins. J Cell Mol Med. 2003; 7: 249-57.

Buytaert E, Dewaele M, Agostinis P. Molecular effectors of multiple cell death pathways initiated by photodynamic therapy. Biochim Biophys Acta. 2007; 1776: 86-107.

Cragg GM, Newman DJ. Plants as a source of anti-cancer agents. J Ethnopharmacol. 2005; 100: 72-79.

Danial NN. BCL-2 family proteins: Critical checkpoints of apoptotic cell death. Clin Cancer Res. 2007; 13: 7254-63.

Elmore S. Apoptosis: A review of programmed cell death. Toxicol Pathol. 2007; 35: 495-516.

Fulda S. Evasion of apoptosis as a cellular stress response in cancer. Int J Cell Biol. 2010; 2010: 370835.

Grana X, Reddy EP. Cell cycle control in mammalian cells: Role of cyclins, cyclin dependent kinases (CDKs), growth suppressor genes and cyclin-dependent kinase inhibitors (CKIs). Oncogene 1995; 11: 211-19.

Hengartner MO. The biochemistry of apoptosis. Nature 2000; 407: 770-76.

Khan M, Rasul A, Yi F, Zhong L, Ma T. Jaceosidin induces p53dependent G2/M phase arrest in U87 glioblastoma cells. Asian Pac J Cancer Prev. 2011; 12: 3235-38.

Khan M, Yu B, Rasul A, Al Shawi A, Yi F, Yang H, Ma T. Jaceosidin Induces Apoptosis in U87 Glioblastoma Cells through G2/M Phase Arrest. Evid Based Complement Alternat Med. 2012; 2012: 703034.

Kim MJ, Han JM, Jin YY, Baek NI, Bang MH, Chung HG, Choi MS, Lee KT, Sok DE, Jeong TS. In vitro anti-oxidant and anti- inflammatory activities of jaceosidin from Artemisia princeps Pampanini cv. Sajabal. Arch Pharm Res. 2008; 31: 429-37.

Kluck RM, Bossy-Wetzel E, Green DR, Newmeyer DD. The release of cytochrome $\mathrm{c}$ from mitochondria: A primary site for Bcl-2 regulation of apoptosis. Science 1997; 275: 1132-36.

Konno S. Effect of various natural products on growth of bladder cancer cells: Two promising mushroom extracts. Altern Med Rev. 2007; 12: 63-68.

Lawen A. Apoptosis-an introduction. Bioessays 2003; 25: 888-96.

Lee JG, Kim JH, Ahn JH, Lee KT, Baek NI, Choi JH. Jaceosidin, isolated from dietary mugwort (Artemisia princeps), induces G2/M cell cycle arrest by inactivating cdc25C-cdc2 via ATMChk1/2 activation. Food Chem Toxicol. 2013; 55: 214-21.

Lee SH, Lee MY, Kang HM, Han DC, Son KH, Yang DC, Sung ND, Lee CW, Kim HM, Kwon BM. Anti-tumor activity of the farnesyl-protein transferase inhibitors arteminolides, isolated from Artemisa. Bioorg Med Chem. 2003; 11: 4545-49.

Leist M, Jaattela M. Four deaths and a funeral: From caspases to alternative mechanisms. Nat Rev Mol Cell Biol. 2001; 2: 58998.

Lv W, Sheng X, Chen T, Xu Q, Xie X. Jaceosidin induces apoptosis in human ovary cancer cells through mitochondrial pathway. J Biomed Biotechnol. 2008; 2008: 394802.

Mallat Z, Tedgui A. Apoptosis in the vasculature: Mechanisms and functional importance. Br J Pharmacol. 2000; 130: 947-62.

Mashima T, Tsuruo T. Defects of the apoptotic pathway as therapeutic target against cancer. Drug Resist Updat. 2005; 8: $339-43$.

Min SW, Kim NJ, Baek NI, Kim DH. Inhibitory effect of eupatilin and jaceosidin isolated from Artemisia princeps on carrageenan-induced inflammation in mice. J Ethnopharmacol. 2009; 125: 497-500.

Ploeg M, Aben KK, Kiemeney LA. The present and future burden of urinary bladder cancer in the world. World J Urol. 2009; 27: 289-93.

Pommier Y, Sordet O, Antony S, Hayward RL, Kohn KW. Apoptosis defects and chemotherapy resistance: Molecular interaction maps and networks. Oncogene 2004; 23: 2934-49.

Rasul A, Bao R, Malhi M, Zhao B, Tsuji I, Li J, Li X. Induction of apoptosis by costunolide in bladder cancer cells is mediated through ROS generation and mitochondrial dysfunction. Molecules 2013; 18: 1418-33.

Rasul A, Ding C, Li X, Khan M, Yi F, Ali M, Ma T. Dracorhodin perchlorate inhibits PI3K/Akt and NF-kappaB activation, upregulates the expression of $\mathrm{p} 53$, and enhances apoptosis. Apoptosis 2012a; 17: 1104-19.

Rasul A, Khan M, Yu B, Ma T, Yang H. Xanthoxyletin, a coumarin induces $S$ phase arrest and apoptosis in human gastric adenocarcinoma SGC-7901 cells. Asian Pac J Cancer Prev. 2011; 12: 1219-23.

Rasul A, Yu B, Khan M, Zhang K, Iqbal F, Ma T, Yang H. Magnolol, a natural compound, induces apoptosis of SGC7901 human gastric adenocarcinoma cells via the mitochondrial and PI3K/Akt signaling pathways. Int J Oncol. 2012b; 40: 1153-61. 
Reed JC. Bcl-2 family proteins. Oncogene 1998; 17: 3225-36.

Reed JC. Apoptosis-based therapies. Nat Rev Drug Discov. 2002; 1: 111-21.

Saha A, Kuzuhara T, Echigo N, Suganuma M, Fujiki H. New role of (-)-epicatechin in enhancing the induction of growth inhibition and apoptosis in human lung cancer cells by curcumin. Cancer Prev Res. 2010; 3: 953-62.

Tan RX, Zheng WF, Tang HQ. Biologically active substances from the genus Artemisia. Planta Med. 1998; 64: 295-302.

Vermeulen K, Van Bockstaele DR, Berneman ZN. The cell cycle:
A review of regulation, deregulation and therapeutic targets in cancer. Cell Prolif. 2003; 36: 131-49.

Wang X. The expanding role of mitochondria in apoptosis. Genes Dev. 2001; 15: 2922-33.

$\mathrm{Wu} \mathrm{XJ}, \mathrm{Hu} \mathrm{Y}$, Lamy E, Mersch-Sundermann V. Apoptosis induction in human lung adenocarcinoma cells by oil-soluble allyl sulfides: Triggers, pathways, and modulators. Environ Mol Mutagen. 2009; 50: 266-75.

Zhang JY. Apoptosis-based anti-cancer drugs. Nat Rev Drug Discov. 2002; 1: 101-02.

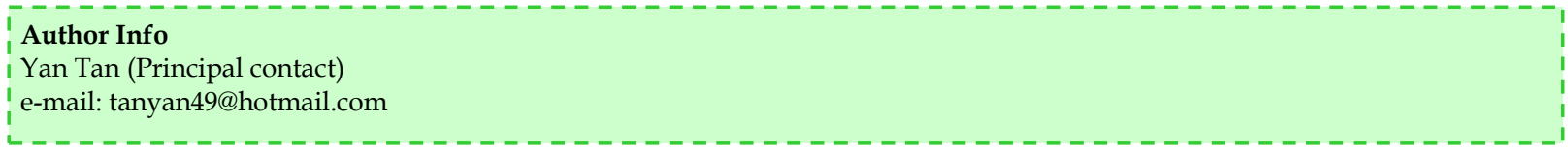

